\title{
Cytomegalovirus colitis in a case of cryptogenic colitis
}

A 78-year-old woman was administered methylprednisolone pulse therapy for 4 days because of exacerbation of bronchial asthma, followed by treatment with prednisolone ( $5 \mathrm{mg}$ ). After 7 days' treatment with prednisolone, the patient developed abdominal pain and continual watery diarrhea. Physical examination revealed tenderness in the upper abdomen. Laboratory data on admission showed severe dehydration (blood urea nitrogen [BUN] $52.5 \mathrm{mg} / \mathrm{dL}$, creatinine $1.78 \mathrm{mg} / \mathrm{dL}$ ) and severe inflammation (C-reactive protein $19.56 \mathrm{mg} / \mathrm{dL}$ ). Sigmoidoscopy showed continuous shallow and girdle ulceration extending from the rectum to the sigmoid colon ( Fig.1). Bacteriologic examination of blood and stool cultures and for Clostridium difficile toxin was negative. Cytomegalovirus (CMV) antigenemia and histological findings including immunohistochemistry were also negative. As treatment with antibiotics had no effect at all, we reevaluated the case for CMV infection. Only real-time polymerase chain reaction (PCR) using colonic biopsy specimens (colonic PCR) was positive for $\mathrm{CMV}$ infection. After antiviral treatment for 2 weeks, the patient's symptoms significantly improved and endoscopy showed healing of the colonic ulceration ( Fig. 2). Hence, a definitive diagnosis of CMV colitis was made.

CMV colitis is an important consideration in patients on immunosuppressive therapies, such as organ transplant recipients and patients with inflammatory bowel disease (IBD) [1]. When its characteristic features, such as deep ulceration, erosions, and mucosal hemorrhage, are observed [2], endoscopy is a useful modality for confirming the diagnoses of CMV infection. In our patient, however, the endoscopic finding of continuous shallow and girdle ulceration extending from the rectum is not typical of CMV colitis. Recently, it has been reported that colonic PCR is useful in diagnosing CMV infection in patients with IBD $[3,4]$. The sensitivity of colonic PCR is higher than that of CMV antigenemia and histological examination including immunohistochemistry $[3,4]$.

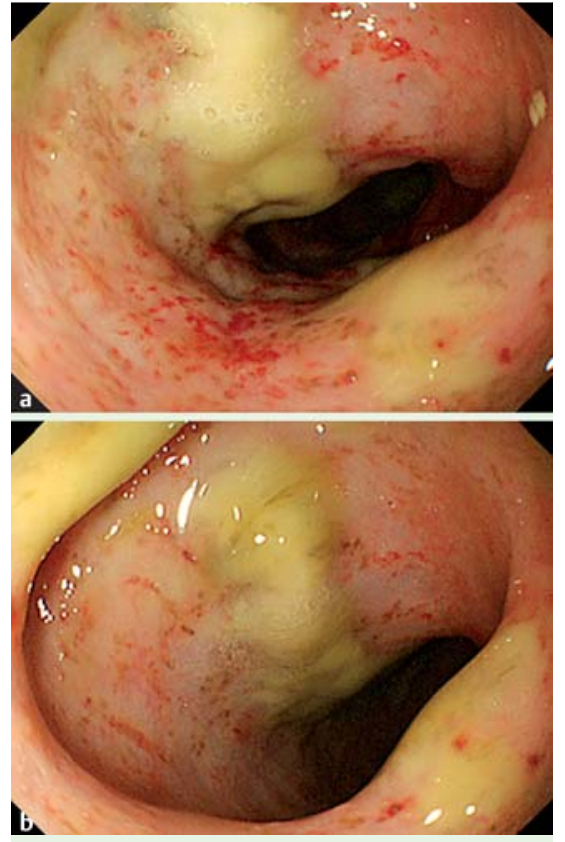

Fig. 1 Sigmoidoscopic views of the sigmoid colon (a) and the rectum (b) in a 78-year-old woman on steroids before antiviral therapy.

CMV infection should always be considered when cryptogenic colitis develops in older patients on any level of immunosuppressive therapies.

Endoscopy_UCTN_Code_CCL_1AD_2AZ

Competing interests: None

\section{T. Yoshino, S. Yazumi, N. Kumagai, A. Yamauchi}

Division of Gastroenterology and Hepatology, Digestive Disease Center, Kitano Hospital, Kitaku, Osaka, Japan

\section{References}

1 Blackman E, Vimadadal S, Nash G. Significance of gastrointestinal cytomegalovirus infection in homosexual males. Am J Gastroenterol 1984; 79: 935-940

2 Goodgame RW. Gastrointestinal cytomegalovirus disease. Ann Intern Med 1993; 119: 924-935

3 Yoshino T, Nakase $H$, Ueno $S$ et al. Usefulness of quantitative real-time PCR assay for early

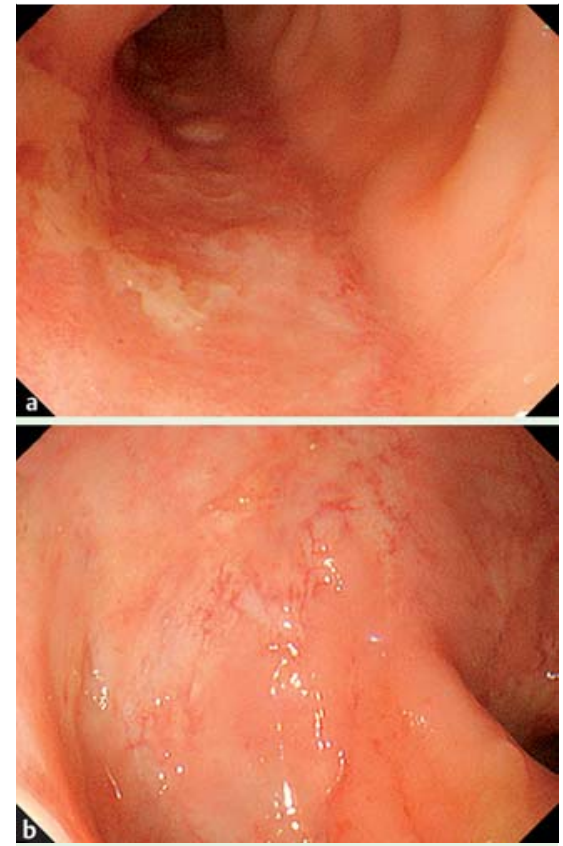

Fig.2 Sigmoidoscopic views of the sigmoid colon (a) and the rectum (b) after antiviral therapy for 2 weeks.

detection of cytomegalovirus infection in patients with ulcerative colitis refractory to immunosuppressive therapies. Inflamm Bowel Dis 2007; 13: 1516-1521

4 Nakase H, Matsumura K, Yoshino T et al. Systematic review: Cytomegalovirus infection in inflammatory bowel disease. J Gastroenterol 2008; 43: $735-740$

Bibliography

Dol http://dx.doi.org/

10.1055/s-0032-1309915

Endoscopy 2012; 44: E346

(c) Georg Thieme Verlag KG

Stuttgart · New York

ISSN 0013-726X

\section{Corresponding author}

\section{S. Yazumi}

Division of Gastroenterology and Hepatology

Digestive Disease Center

Kitano Hospital

2-4-20 Ohgimachi

Kitaku

Osaka 530-8480

Japan

Fax: +81-6-6361-0588

s-yazumi@kitano-hp.or.jp 